\title{
Análise da força articular resultante entre diferentes movimentos do ombro com e sem carga: estudo preliminar
}

\author{
Daniel Cury Ribeiro 1,2 \\ Jefferson Fagundes Loss 1 \\ Joelly Mahnic de Toledo ${ }^{1}$ \\ Marcelo Peduzzi de Castro ${ }^{1}$ \\ Fábia Milman Krumholz ${ }^{1}$ \\ Felipe de Osório Marques 1
}

https://doi.org/10.5628/rpcd.10.01.129

\author{
${ }^{1}$ Universidade Federal do Rio Grande do Sul \\ Escola Superior de Educação Física \\ Porto Alegre - RS \\ Brasil \\ ${ }^{2}$ Faculdade da Serra Gaúcha \\ Escola de Educação e Saúde \\ Caxias do Sul - RS \\ Brasil
}

\section{RESUMO}

Os modelos de segmentos rígidos são extremamente úteis para auxiliar a compreensão do tipo de sobrecarga que as articulações estão expostas. O objetivo deste trabalho foi analisar a força articular resultante e momento proximal durante atividades sem e com carga externa, em um estudo preliminar. Um indivíduo realizou flexão, extensão, abdução de ombro e flexão de cotovelo (sem e com carga). Dados cinemáticos foram obtidos por videogrametria (freqüência de amostragem de $50 \mathrm{cam}$ pos/s). Um modelo de segmentos rígidos foi utilizado para obtenção dos dados cinéticos. O modelo é regido pelas equações de movimento de Newton-Euler. Os resultados encontrados evidenciaram aumentos não proporcionais na força e momento resultante. A força resultante longitudinal foi a única que apresentou aumento proporcional frente à carga externa. As componentes da força de cisalhamento e momento proximal apresentaram aumentos aleatórios. O aumento da carga externa promoveu aumentos da força e momento proximal, porém não de forma proporcional. As componentes de força e momento resultante sofrem influência da carga externa de maneira independente. Isto evidencia a necessidade de um controle rígido sobre os exercícios prescritos para diferentes disfunções do membro superior.

Palavras-chave: ombro, força, cinética, torque

\begin{abstract}
Analysis of the joint reaction forces during different shoulder movements with and without external load: a preliminary study

Link segment models are extremely useful for increasing the comprehension of joint overload. The aim of the present study was to analyze proximal joint reaction forces and moments during different movements performed with and without external load. One subject performed shoulder flexion, extension and abduction, and elbow flexion movements (with and without external load) Kinematic data were obtained by videogrammetry (frequency sample 50 fields/s). One link segment model was used to obtain kinetic data. The model is governed by Newton/Euler movement equations. The results suggested a not proportional increasing of proximal joint reaction forces and moments. The proximal joint reaction force longitudinal component was the only one that increased proportionally to the external load. Proximal joint reaction force shearing components and proximal moments presented increasing values of different magnitudes. The use of external load promoted increased magnitudes of proximal joint reaction force and moment, although it was not proportional. Proximal joint reaction force and moment are influenced in different ways by the external load. This suggests the need of a strict control of the prescribed exercises for different shoulder dysfunctions.
\end{abstract}

Key-words: shoulder, force, kinetics, torque 


\section{INTRODUÇÃO}

Modelos biomecânicos têm sido comummente utilizados com o objectivo de estimar as forças de reacção proximal (FRP) e momento proximal (MP) tanto durante actividades esportivas como durante actividades de vida diária (AVDs) $5,7,8,17,26,28,29)$. A estrutura dos modelos pode variar, conforme o objectivo do estudo. Alguns estudos utilizam modelos músculo-esqueléticos $(15,21,22,28)$ enquanto outros modelos utilizam estruturas mais simples, os denominados modelos de segmentos rígidos $(5,8,16,19)$.

Os modelos de segmentos rígidos, apesar de operarem com forças e momentos resultantes, são extremamente úteis para auxiliar a compreensão do tipo de sobrecarga a que as articulações estão expostas $(7$, $8,13)$. Recentemente, um estudo destacou a necessidade de estruturar um banco de dados normativos sobre as forças articulares resultantes do membro superior durante AVDs. Os autores destacam a importância deste tipo de estudo para melhor compreensão da sobrecarga a que as articulações do membro superior estão expostas ${ }^{(13)}$. Nos últimos anos, alguns estudos voltaram-se para análise do efeito de uma carga externa sobre o ritmo escapuloumeral durante a elevação do braço(4, 10, 11, 14, 20). Outros, voltaram-se para a análise de gestos esporti-

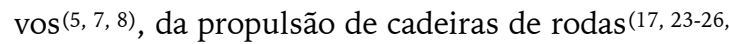
29) e de actividades de vida diária $(9,13,28)$. Ainda, durante $05^{\text {th }}$ Meeting of the International Shoulder Group foram apresentados dados comparando o número de publicações referentes ao membro superior e membro inferior. A razão pela qual existe um menor número de publicações relacionadas ao membro superior não é evidente, no entanto, é claro a necessidade de mais publicações vinculadas ao membro superior(27). Não encontramos estudos que analisassem os efeitos da carga externa (halter) nas forças resultantes do ombro. Assim, o objectivo deste trabalho é analisar a força articular resultante e momento proximal durante actividades sem e com carga externa.

\section{MATERIAIS E MÉTODOS}

O estudo contou com a participação de um indivíduo do sexo masculino, de idade de 25 anos, $1,79 \mathrm{~m}$ de altura e $80 \mathrm{~kg}$. Antes de qualquer envolvimento com este trabalho, o indivíduo assinou o Termo de
Consentimento Informado, consentindo formalmente na participação desta pesquisa. A amostra foi de quatro gestos comummente realizados durante programas de reforço muscular do membro superior. Os gestos avaliados foram: flexão, extensão, abdução de ombro e flexão de cotovelo. Estes gestos foram realizados sem carga externa e com uma carga equivalente a $5 \%$ do peso corporal (PC) do indivíduo.

A aquisição dos dados cinemáticos foi feita através de cinco câmaras de vídeo (JVC GR-DVL9800). A frequência de amostragem foi de 50 campos/s, com um tempo de abertura das câmeras (shutter) ajustado em $1 / 250$. No suporte de cada câmera, foram fixados um iluminador com mesma direção óptica da câmera para aumentar o contraste entre o marcador reflexivo e o restante da imagem. O software Dvideow foi utilizado para digitalização das imagens e reconstrução espacial(6). Foi utilizado o modo semi-automático de digitalização das imagens, com os seguintes algoritmos: inversão, erosão, getmarkers(2). A reconstrução espacial foi feita através do método direct linear transformation (DLT) ${ }^{(1)}$. O sincronismo intracameras foi realizado através do canal de áudio(3). A acurácia da medida entre dois pontos foi assumida como representativa do erro de medida do sistema ${ }^{(2,}$ 3). Dois marcadores reflexivos foram fixados em uma barra rígida, a qual foi deslocada dentro do volume de calibração, de aproximadamente, 1,262 x 1,082 x $0,902 \mathrm{~m}^{3}$. A medida entre os marcadores $(231,50$ $\mathrm{mm}$ ) foi medida através de um paquímetro (resolução de $0,05 \mathrm{~mm}$ ), a qual foi assumida como valor real. A acurácia foi, então calculada através da seguinte equação:

$$
a=\sqrt{\left(b^{2}+p^{2}\right)}
$$

Em que:

$a=$ acurácia;

$b=$ bias do sistema, dada pela diferença entre a média dos valores (medidas experimentais) e a medida real (medida directa); $p^{2}=$ precisão do sistema, dado pelo desvio padrão da média das medidas experimentais.

A referências anatómicas de interesse foram determinadas através do uso de marcadores retroreflexivos em formato de esfera, com $15 \mathrm{~mm}$ de diâmetro. As referências anatómicas utilizadas foram: processo 
espinhoso da sétima vértebra cervical (C7); processo espinhoso da oitava vértebra dorsal (T8); processo coracóide (PC); incisura jugular (IJ); processo xifóide (PX); articulação esterno-clavicular (SC); articulação acrômio-clavicular (AC); ângulo medial da escápula (AM); ângulo inferior da escápula (AI); ângulo acromial (AA); centro de rotação da glenoumeral (GU) o qual foi estimado pelo método de regressão linear(12); epicôndilo lateral (EL); epicôndilo medial (EM); processo estilóide rádio (PER); e processo estilóide ulna (PEU), conforme recomendações da International Society of Biomechanics(31).

Em virtude da interferência dos músculos do antebraço no posicionamento dos marcadores reflexivos do EL e EM, foi utilizado um marcador técnico (MT) posicionado no segmento do braço(18). O MT consiste de uma haste em formato de " $\mathrm{T}$ ", contendo três marcadores retroreflexivos não colineares. Um sistema de coordenada para cada segmento corporal (mão, antebraço, braço, escápula e tronco) foi definido. A definição destes sistemas, bem como da descrição cinemática respeitou as recomendações da International Society of Biomechanics(31). As sequências de rotação, assim como o segmento proximal utilizado como referência estão descritos na Tabela 1.

Tabela 1. Sequência de rotação adoptada para cada segmento corporal. SCG = Sistema de Coordenada Global, $S C L=$ Sistema de Coordenada Local. $X, Y, Z$ [maiúsculas] referem-se aos eixos do sistema de coordenada de referência. $x, y, z$ (minúsculas) referem-se aos eixos do SCL do segmento distal.

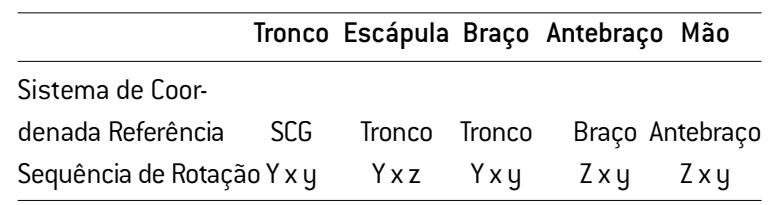

O modelo utilizado no presente trabalho é regido pelas equações de movimento linear e angular de Newton-Euler (equações 2 e 3):

Em que:

$$
\begin{aligned}
& \sum \mathbf{F}=m \mathbf{a}_{\mathrm{cm}} \\
& \sum \mathbf{M}=\dot{\mathbf{H}}
\end{aligned}
$$

$\mathrm{SF}=$ somatório das forças (vetorial);

$m=$ massa;

$\mathrm{a}_{\mathrm{cm}}=$ aceleração linear do centro de massa;

$\mathrm{SM}=$ somatório dos momentos (vetorial);

$\dot{\mathrm{H}}=$ taxa de variação do momento de angular;
O modelo utiliza os dados cinemáticos, cinéticos e antropométricos como variáveis independentes. As variáveis dependentes são as Frp e Mp envolvidos. Maiores detalhes sobre o modelo podem ser encontrados na literatura(16).

Os dados cinemáticos foram filtrados com filtro digital passa-baixa Butterworth, ordem 3, sendo a frequência de corte determinada pelo do método da Análise Residual, proposto por Winter(30). Por meio deste método, foi determinado um valor de frequência de corte para cada coordenada espacial global (X, Y, Z) de cada referência anatómica, em cada gesto realizado. As frequências de corte variaram de 0,5 a 4,9 Hz. A coleta de dados foi realizada em 2 etapas (sem e com carga). Cada uma destas etapas era dividida em dois estágios (registo em vídeo estático e dinâmico). Durante o registo em vídeo estático, o indivíduo se encontrava utilizando marcadores reflexivos em todas referências anatómicas e ainda, utilizava o marcador técnico. Este estágio permite a posterior dedução da localização dos pontos anatómicos EL e EM. Durante o registo em vídeo dinâmico, o indivíduo se encontrava utilizando todos marcadores com excepção daqueles do EL e EM.

$\mathrm{O}$ indivíduo realizou cinco repetições para cada gesto motor, primeiramente sem carga e, posteriormente, com uma carga equivalente à $5 \%$ do seu PC. Os gestos analisados foram: flexão de ombro, extensão de ombro, abdução de ombro e flexão de cotovelo.

Todos estes gestos foram realizados com uma velocidade angular média de $45^{\circ}$ s, a qual foi acompanhada por um metrónomo.

Para verificar a interferência da carga na execução do gesto, foi avaliada a reprodutibilidade dos ângulos articulares do ombro (plano de elevação, ângulo de elevação e ângulo de rotação interna e externa) para cada gesto com e sem carga. A reprodutibilidade também foi avaliada para os ângulos articulares do cotovelo (ângulo de flexão, ângulo de carregamento e ângulo de pronação-supinação). A reprodutibilidade foi expressa quantitativamente através do Erro Rms, assumida como sendo a diferença da raiz quadrada entre todos os pontos coletados pareados nas duas etapas (sem e com carga) (13), conforme a equação:

$$
\text { Erro Rms }=\frac{\sqrt{\sum_{i} 1000\left(x 1_{i}-x 2_{i}\right)^{2}}}{2000}
$$


Em que:

$i=1,2,3,4, \ldots \mathrm{n}$;

$x 1=$ dados de cada variável analisada da etapa 1 ;

$x 2=$ dados de cada variável analisada da etapa 2;

\section{RESULTADOS}

Os valores de acurácia, bias e precisão do sistema de videogrametria foram, em média, de $1,7 \mathrm{~mm}, 0,5$ $\mathrm{mm}$, e 1,6 mm, respectivamente. Foram encontrados baixos valores de Erro Rms para cada gesto avaliado (Tabela 2). O maior valor de Erro Rms foi encontrado para o movimento de extensão do ombro (sem e com carga): 23,11graus (para o ângulo do plano de elevação do ombro).

Tabela 2. Erro Rms para cada ângulo de movimento articular (ombro e cotovelo] entre os gestos sem e com carga.

\begin{tabular}{lccccc}
\hline \multicolumn{5}{c}{ Movimento } \\
\hline \multicolumn{5}{c}{ Ombro } & Cotovelo \\
\hline Erro Rms & R1 & 10,61 & 11,76 & 23,11 & 15,56 \\
(graus) & R2 & 6,08 & 7,02 & 8,57 & 1,59 \\
& R3 & 6,07 & 5,30 & 5,33 & 1,61 \\
\hline
\end{tabular}

R1: plano de elevação (para o ombro) e ângulo de flexão para o cotovelo; R2: ângulo de elevação (para o braço) e ângulo de carregamento para o cotovelo;R3: ângulo de rotação interna/externa (para o ombro] e ângulo de prono-supinação para o cotovelo (rádio-ulnar).

Os resultados de pico de ângulo, FRP e MP para a articulação do ombro (gestos de abdução, flexão, extensão) e cotovelo (flexão de cotovelo) estão descritos na Tabela 3. Nesta tabela, estão descritos os valores de pico angular para cada movimento articular, bem como o valor de pico de FRP e MP para cada eixo do sistema de coordenada local do segmento braço e antebraço. Os valores de FRP e MP foram maiores durante os gestos realizados com carga externa, como esperado.

A Tabela 4 apresenta a razão entre os valores de pico para cada ângulo articular, e pico de FRP e MP entre os gestos realizados com carga e sem carga. Esta tabela permite verificar o aumento do pico de cada variável analisada (ângulo, FRP e MP) quando realizado o gesto com carga externa. Para os valores de ângulo, praticamente todos os movimentos articulares atingiram picos angulares similares (todas
Tabela 3. Valores de pico para cada ângulo de articular (ombro e cotovelo] e cada componente de FRP e MP para os segmentos do braço e antebraço durante os gestos analisados

\begin{tabular}{|c|c|c|c|c|}
\hline \multicolumn{5}{|c|}{ Movimento } \\
\hline & & Ombro & & Cotovelo \\
\hline & Abdução & Flexão & Extensão & Flexão \\
\hline $\mathrm{R} 1$ & 10,24 & 86,46 & $-68,79$ & 143,08 \\
\hline $\mathrm{R} 2$ & $-88,54$ & $-96,64$ & $-47,59$ & 0,70 \\
\hline R3 & 19,10 & 28,65 & 38,08 & 14,59 \\
\hline$x$ & 13,83 & 26,58 & $-4,52$ & 17,20 \\
\hline y & 38,03 & 40,76 & 38,88 & 16,58 \\
\hline z & 38,08 & 28,69 & 8,56 & 7,00 \\
\hline$x$ & $-10,33$ & $-7,22$ & $-2,47$ & $-1,17$ \\
\hline$y$ & $-0,26$ & $-0,17$ & $-0,23$ & 0,06 \\
\hline z & 13,89 & 6,76 & $-0,41$ & 2,79 \\
\hline $\mathrm{R} 1$ & 1,27 & 74,27 & $-89,99$ & 144,21 \\
\hline $\mathrm{R} 2$ & $-101,76$ & $-95,99$ & $-49,40$ & 3,97 \\
\hline R3 & 28,66 & 37,02 & 35,49 & 13,25 \\
\hline$x$ & 72,96 & 46,59 & $-14,91$ & 63,98 \\
\hline$y$ & 88,32 & 87,07 & 86,17 & 65,08 \\
\hline z & 64,19 & 79,01 & 40,57 & 26,56 \\
\hline$x$ & $-26,82$ & $-33,22$ & $-18,08$ & $-6,92$ \\
\hline y & $-0,68$ & $-0,75$ & 1,87 & 0,46 \\
\hline z & 32,76 & 21,30 & $-0,35$ & 16,20 \\
\hline
\end{tabular}

R1: plano de elevação para o ombro e ângulo de flexão para o cotovelo;R2: ângulo de elevação para o braço e ângulo de carregamento para o cotovelo;R3: ângulo de rotação interna/externa para o ombro e ângulo de prono-supinação para o cotovelo (rádio-ulnar).FRP: força de reação proximal; MP: momento proximal

razões próximas de 1,0). As excepções foram: plano de elevação (razão de 0,12 ) durante o movimento de abdução do ombro e ângulo de carregamento (razão de 5,70).

As razões de pico (carga/sem carga) para a FRP e MP variaram. O menor e maior valor de razão de pico para FRP foi de 1,69 e 5,28, respectivamente (Tabela 4). Já o menor valor (em módulo) de razão de pico para MP foi de 0,86 e o maior de 8,42. 
Tabela 4. Razões de Pico (Carga/Sem carga) para os diferentes gestos analisados.

\begin{tabular}{lccccc}
\hline \multicolumn{5}{c}{ Movimento } \\
\hline & & \multicolumn{5}{c}{ Ombro } & & Cotovelo \\
\hline \multirow{2}{*}{ Ángulo (graus) } & R1 & 0,12 & 0,86 & 1,31 & 1,01 \\
& R2 & 1,15 & 0,99 & 1,04 & 5,70 \\
& R3 & 1,50 & 1,29 & 0,93 & 0,91 \\
FRP & x & 5,28 & 1,75 & 3,30 & 3,72 \\
& y & 2,32 & 2,14 & 2,22 & 3,92 \\
MP & z & 1,69 & 2,75 & 4,74 & 3,80 \\
& x & 2,60 & 4,60 & 7,33 & 5,93 \\
& y & 2,62 & 4,39 & $-8,14$ & 7,65 \\
& z & 8,42 & 3,15 & 0,86 & 5,80 \\
\hline
\end{tabular}

R1: plano de elevação para o ombro e ângulo de flexão para o cotovelo; $R 2$ : ângulo de elevação para o braço e ângulo de carregamento para o cotovelo; $R 3$ : ângulo de rotação interna/externa para o ombro e ângulo de prono-supinação para o cotovelo (rádio-ulnar).FRP: força de reação proximal; MP: momento proximal

Os comportamentos de FRP e MP de cada componente $(x, y, z)$ apresentam variações, na medida em que a amplitude de movimento se altera.

Evidentemente que, conforme o gesto analisado, o comportamento das componentes, bem como suas magnitudes, é diferente. As Figuras 1 e 2 apresentam o comportamento da FRP durante a abdução de ombro sem e com carga, respectivamente.

$$
\cdot x \cdot y-z
$$

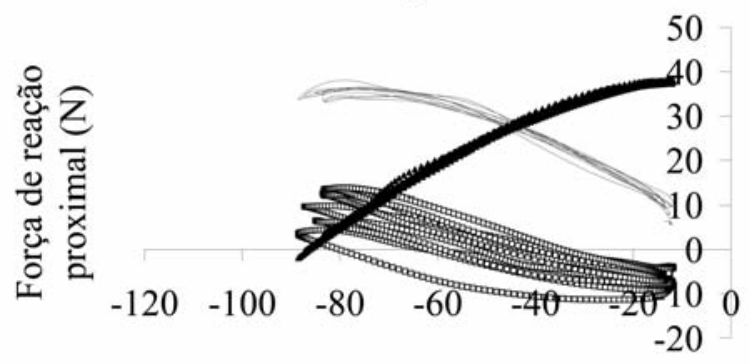

Ângulo de elevação (graus)

Figura 1. Força de reação proximal durante abdução do ombro sem carga.
Com o incremento da carga, o comportamento da componente $x$ (anteroposterior) torna-se distinto em relação ao seu comportamento durante o gesto sem carga. Estes dados evidenciam um deslocamento posterior da cabeça do úmero em relação à glenoide e uma força de estabilização por parte do manguito rotador e estruturas cápsulo-ligamentares no sentido de anteriorização da cabeça do úmero. Além disso, evidentemente, todas componentes da FRP apresentam maiores magnitudes.

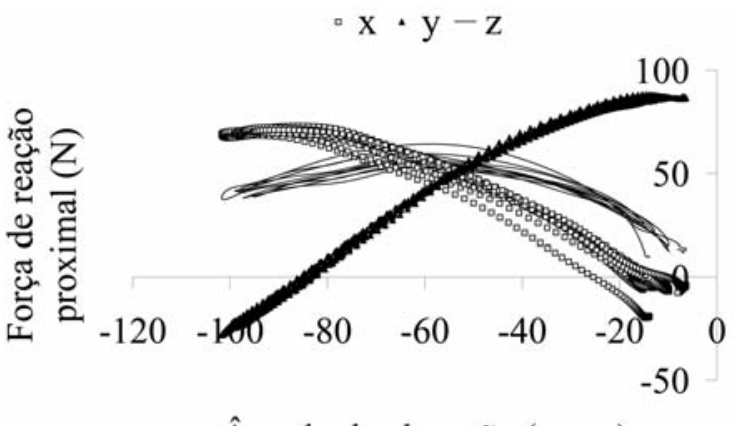

Ângulo de elevação (graus)

Figura 2. Força de reação proximal durante abdução do ombro com carga.

O comportamento das componentes do MP é similar entre os gestos com e sem carga, com excepção da componente $\mathrm{z}$, a qual adopta um comportamento mais padronizado durante a execução do gesto com carga (Figuras 3 e 4). Através destes resultados, fica explícito a magnitude de sobrecarga sobre as estruturas músculo-tendíneas dos músculos abdutores e flexores de ombro, que conjuntamente, promovem o movimento de elevação no plano escapular.

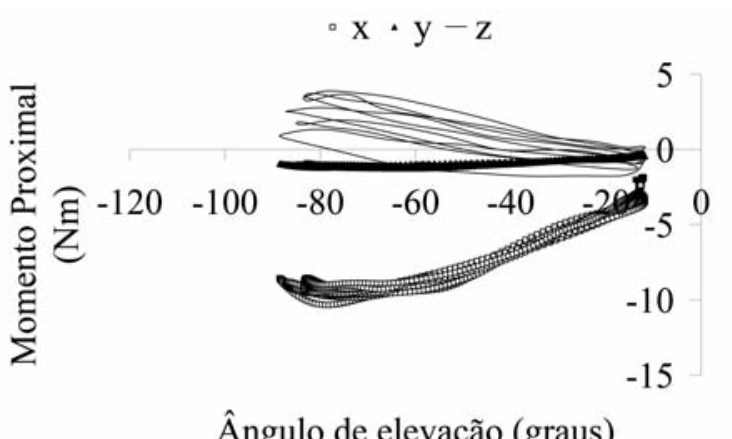

Figura 3. Momento proximal durante abdução do ombro sem carga. 


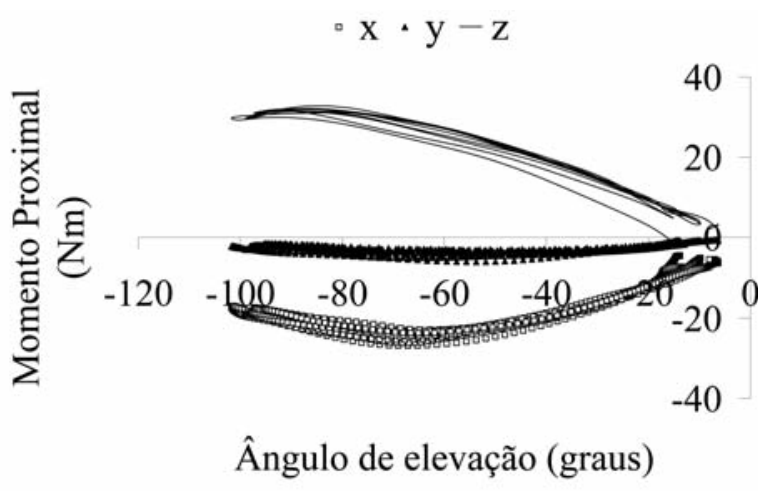

Figura 4. Momento proximal durante abdução do ombro com carga.

\section{DISCUSSÃO}

A maior parte da literatura apresenta dados referentes a gestos esportivos como arremessos $(5,7,8)$,

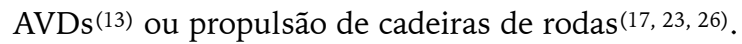
Não encontramos nenhum estudo voltado para atividades comumente realizadas no treinamento de força, ou exercícios de reabilitação.

Fleisig et al.(7) identificaram que as amplitudes de movimento com maior risco lesivo correspondiam àquelas em que ocorreram os picos de FRP e MP. Estes autores avaliaram o gesto de arremesso do beisebol. Os picos de MP foram de $67 \mathrm{Nm}$ (para rotação interna), $64 \mathrm{Nm}$ (para pronação de cotovelo) e 97 $\mathrm{Nm}$ (para abdução horizontal). Com relação aos picos de FRP, os valores encontrados foram: $1.090 \mathrm{~N}$ de força compressiva e $400 \mathrm{~N}$ de força posterior. Hong et al.(8) avaliaram o movimento do arremesso no beisebol, com objectivo identificar o padrão de comportamento do MP do tronco, ombro e cotovelo. O pico do MP de abdução no ombro atingiu valores próximos de $300 \mathrm{Nm}$. Feltner e Taylor(5) avaliaram os valores de MP e FPR durante o arremesso do pólo aquático. Próximos ao instante do arremesso, os momentos de abdução, rotação interna e flexão horizontal atingiram, em média, magnitudes próximas de $60 \mathrm{Nm}$. Os estudos citados acima apresentam valores de MP muito acima daqueles encontrados no presente estudo. No entanto, os gestos analisados são balísticos, o que interfere de forma muito clara na magnitude da FRP e do MP. Os resultados do presente estudo apresentaram razões de pico de força próximas de 2,0 (no eixo y - longitudinal - do braço) para os movimentos do ombro (flexão, abdução e extensão). Isto ocorreu, pois a FRP durante os movimentos sem carga corresponde à força gravitacional e inercial do segmento do braço(16, 32). Na medida em que o gesto foi executado em baixa velocidade, a força inercial é mínima, enquanto que a força gravitacional corresponde, aproximadamente, a $5 \%$ do peso corporal. A força externa aplicada, nos gestos com carga, foi de $40 \mathrm{~N}$, a qual também equivale a $5 \%$ do PC. Assim, o pico da FRP longitudinal dobrou para o segmento do braço. No entanto, é importante destacar que, para as outras componentes x e $z$, isso não ocorreu (Tabela 4). Provavelmente, este é o dado que mereça mais atenção no presente trabalho, pois isso demonstra que o pico de FRP não corresponde, necessariamente, a um aumento proporcional em relação à carga aplicada. Isto tem especial relevância na reabilitação de disfunções articulares do membro superior.

$\mathrm{O}$ acréscimo da magnitude do MP em suas diferentes componentes se deve, exclusivamente, à orientação do segmento no espaço. Na medida em que os gestos foram realizados em velocidades baixas e constantes (foram monitoradas por metrónomo), o MP representa a actividade muscular dos agonistas do movimento(16, 30, 32). Assim, o aumento da carga externa é acompanhando por um aumento do momento produzido pelos músculos do ombro. Três razões de pico do MP merecem especial atenção: a razão de pico do eixo y durante a extensão de ombro, a razão de pico do eixo $z$ durante a abdução de ombro e a razão de pico em torno do eixo y durante a flexão de cotovelo. Estas apresentaram valores de razão de pico próximos de 8,0. No entanto, o pico de MP para estes eixos, durante os gestos sem e com carga, foram muito baixos (Tabela 3), não representando aumentos do MP consideráveis.

A realização dos gestos com carga apresentou picos de ADM muito similares aos gestos realizados sem carga (Tabelas 2 e 4). Os valores de Erro Rms foram, para maior parte dos gestos, bastante baixos entre os gestos sem e com carga. Valores de Erro Rms maiores ficaram por conta da R1 para os movimentos de flexão e extensão do ombro. Isso sugere que o padrão de execução do movimento não foi substancialmente alterado com a inserção da carga externa de 5\% do PC. Além disso, razões de pico angulares próximos de 1,0 
foram encontradas para praticamente todos os gestos. A exceção foi o ângulo de carregamento do cotovelo, o qual, sem carga, atingiu um pico de 0,70 grau e, com carga, 3,97 graus. Apesar de a razão de pico ter sido grande, a variação em termos absolutos foi muito pequena, visto que o Erro Rms foi de 1,59 graus. Este estudo envolveu a análise de gestos realizados por apenas um indivíduo. No entanto, apresenta resultados importantes sobre o padrão de comportamento e aumento das FRP e MP. É apresentado um método para quantificação e análise das forças articulares e momentos proximais. Os resultados do presente estudo reforçam conclusões de outros estudos, os quais afirmam a necessidade de se estabelecer uma base de dados normativos cinemáticos e cinéticos, para actividades realizadas com os membros superiores $(9,13,27)$.

Os resultados sugerem que o aumento da carga externa equivalente a $5 \%$ do PC foi suficiente para acarretar em aumentos na magnitude da força de reacção proximal e momento proximal do ombro e cotovelo. No entanto, o dado mais relevante é, justamente, o aumento aleatório destas forças e momentos. Não encontramos aumentos proporcionais da força e momento proximal para todos componentes de força e momento proximal. Além disso, a execução do gesto não acarretou em variação expressiva do padrão de movimento articular.

\section{AGRADECIMENTOS}

Este estudo recebeu apoio financeiro da Coordenação de Aperfeiçoamento de Pessoal de Nível Superior (CAPES) - Brasil.

\section{CORRESPONDÊNCIA}

Daniel Cury Ribeiro

Endereço: Rua Barão do amazonas, 793/401

CEP: 90670-003

Porto Alegre - RS, Brasil.

E-mail: daniel.cury.ribeiro@gmail.com 


\section{REFERÊNCIAS}

1. Abdel-Aziz, e Karara. (1971). Direct linear transformation from comparator coordinates into objects space coordinates in close-range photogrammetry. Proceedings of the Symposium on Close-Range Photogrammetry, 1-18.

2. Andrade LM, Araújo AGN, Barros RML (2004). Análise de marcha: protocolo experimental para posicionamento e orientação dos segmentos do corpo humano baseado em sistemas de marcas técnicas. Brazilian Journal of Biomechanics, 9: 33-40

3. Barros RML, Russomanno TG, Brenzikover R, Figueroa PJ (2006). A method to synchronise video cameras using the audio band. Journal of Biomechanics, 39: 776-780

4. Ebaugh DD, McClure PW, Karduna AR (2005). Three-dimensional scapulothoracic motion during active and passive arm elevation. Clin Biomech (Bristol, Avon), 20(7): 700-709

5. Feltner ME, Taylor G (1997). Three-Dimensional kinetic of the shoulder, elbow, and wrist during a penalty throw in water polo. Journal of Applied Biomechanics, 13: 347-372

6. Figueroa PJ, Leite NJ, Barros RM (2003). A flexible software for tracking of markers used in human motion analysis. Comput Methods Programs Biomed, 72(2): 155-165

7. Fleisig GS, Andrews JR, Dillman CJ, Escamilla RF (1995). Kinetics of baseball pitching with implications about injury mechanicsms. American Journal of Sports Medicine, 23(2): 233-239

8. Hong DA, Cheung TK, Roberts EM (2001). A three-dimensional, six-segment chain analysis of forceful overarm throwing. J Electromyogr Kinesiol, 11(2): 95-112

9. Magermans DJ, Chadwick EK, Veeger HE, van der Helm FC (2005). Requirements for upper extremity motions during activities of daily living. Clin Biomech (Bristol, Avon), 20(6): 591-599

10. McClure PW, Michener LA, Sennett BJ, Karduna AR (2001). Direct 3-dimensional measurement of scapular kinematics during dynamic movements in vivo. J Shoulder Elbow Surg, 10(3): 269-277

11. McQuade KJ, Dawson J, Smidt GL (1998). Scapulothoracic muscle fatigue associated with alterations in scapulohumeral rhythm kinematics during maximum resistive shoulder elevation. J Orthop Sports Phys Ther, 28(2): 74-80

12. Meskers CG, van der Helm FC, Rozendaal LA, Rozing PM (1998). In vivo estimation of the glenohumeral joint rotation center from scapular bony landmarks by linear regression. J Biomech, 31(1): 93-96

13. Murray IA, Johnson GR (2004). A study of the external forces and moments at the shoulder and elbow while performing every day tasks. Clin Biomech (Bristol, Avon), 19(6): 586-594

14. Pascoal AG (2001). Ombro e Elevação do Braço. Análise cinemática e eletromiográfica sobre a influência da carga externa e velocidade do braço no ritmo escápulo-umeral. Tese de Doutorado. Universidade Técnica de Lisboa, Lisboa.

15. Praagman M, Stokdijk M, Veeger HE, Visser B (2000). Predicting mechanical load of the glenohumeral joint, using net joint moments. Clin Biomech (Bristol, Avon), 15(5): 315-321

16. Ribeiro DC (2006). Implementação de um modelo para cálculo das forças proximais e momentos proximais resultantes para $o$ membro superior. Mestrado, Universidade Federal do Rio Grande do Sul, Porto Alegre.

17. Rodgers MM, Tummarakota S, Lieh J (1998). ThreeDimensional Dynamic Analysis of Wheelchair Propulsion. J Apllied Biomech, 14: 80-92

18. Schmidt R, Disselhorst-Klug C, Silny J, Rau G (1999). A marker-based measurement procedure for unconstrained wrist and elbow motions. J Biomech, 32(6): 615-621

19. Sogaard K, Laursen B, Jensen BR, Sjogaard G (2001). Dynamic loads on the upper extremities during two different floor cleaning methods. Clin Biomech (Bristol, Avon), 16(10): 866-879

20. Tsai NT, McClure PW, Karduna AR (2003). Effects of muscle fatigue on 3-dimensional scapular kinematics. Arch Phys Med Rehabil, 84(7): 1000-1005

21. van der Helm FC (1994). A finite element musculoskeletal model of the shoulder mechanism. J Biomech, 27(5): 551569

22. van der Helm, F. C. (1994). Analysis of the kinematic and dynamic behavior of the shoulder mechanism. J Biomech, 27(5): 527-550

23. van der Helm FC, Veeger HE (1996). Quasi-static analysis of muscle forces in the shoulder mechanism during wheelchair propulsion. J Biomech, 29(1): 39-52

24. van der Woude LH, van Kranen E, Ariens G, Rozendal RH, Veeger HE (1995). Physical strain and mechanical efficiency in hubcrank and handrim wheelchair propulsion. J Med Eng Technol, 19(4): 123-131

25. van Drongelen $S$, de Groot S, Veeger HE, Angenot EL, Dallmeijer AJ, Post, MW, and van der Woude LH (2006). Upper extremity musculoskeletal pain during and after rehabilitation in wheelchair-using persons with a spinal cord injury. Spinal Cord, 44(3): 152-159

26. Van Drongelen S, Van der Woude LH, Janssen TW, Angenot EL, Chadwick EK, Veeger DH (2005). Mechanical load on the upper extremity during wheelchair activities. Arch Phys Med Rehabil, 86(6): 1214-1220

27. Veeger DJ, Pascoal AG (2006). Upper extremity biomechanics: Are we closing the gap? Proceedings of the 5 th meeting of the International Shoulder Group. Clinical Biomechanics, 21: (S1-S2)

28. Veeger HE, Magermans DJ, Nagels J, Chadwick EK, and van der Helm FC (2006). A kinematical analysis of the shoulder after arthroplasty during a hair combing task. Clin Biomech (Bristol, Avon), 21 (Suppl 1): S39-S44

29. Veeger HE, Rozendaal LA, van der Helm FC (2002). Load on the shoulder in low intensity wheelchair propulsion. Clin Biomech (Bristol, Avon), 17(3): 211-218

30. Winter DA (2005). Biomechanics and motor control of human movement, 3rd Ed., Wiley, Hoboken, NJ

31. Wu G, van der Helm FC, Veeger HE, Makhsous M, Van Roy P, Anglin C, Nagels J, Karduna AR, McQuade K, Wang X, Werner FW, Buchholz B (2005). ISB recommendation on definitions of joint coordinate systems of various joints for the reporting of human joint motion-Part II: shoulder, elbow, wrist and hand. J Biomech, 38(5): 981-992

32. Zatsiorsky VM (2002). Kinetics of human motion, Human Kinetics, Champaign: IL 